\title{
Effects of Lower Extremity Eccentric-Based Training on Muscle Strength and Physical Function in Older Adults: A Randomized Controlled Pilot Trial
}

\author{
Dae Young Kim ${ }^{1,3}$, Seung Lyul Oh ${ }^{2,3}$, Wook Song ${ }^{1,2}$, Jae-Young Lim ${ }^{2,3}$ \\ ${ }^{1}$ Health and Exercise Science Laboratory, Institute of Sports Science, Seoul National University, Seoul; ${ }^{2}$ Institute on Aging, Seoul National University, Seoul; ${ }^{3} \mathrm{Aging}$ and \\ Mobility Biophysics Laboratory, Seoul National University Bundang Hospital, Seongnam, Korea
}

PURPOSE: Reduced muscular strength is a key player in loss of physical function and quality of life in older adults. It has been reported that eccentric training has positive effects on the preservation of eccentric strength, produces less delayed onset muscle soreness, and has a lower metabolic cost for older individuals. Therefore, the present study investigated the effects of eccentric-based training on skeletal muscle strength and physical function in older adults.

METHODS: Sixteen healthy older subjects (over the age of 65) participated either in eccentric training group (ETG, $\mathrm{n}=8$ ) or in conventional resistance training group $(\mathrm{CTG}, \mathrm{n}=8$ ) twice a week for eight weeks. The ETG group performed motorized eccentric training, and the CTG group performed a leg press using air pressure. Physical function and muscular strength assessments were performed before and after 8 weeks. The primary outcomes for physical function included gait speed, stair climbing, and the chair stand test, and the secondary outcomes included muscle strength and power.

RESULTS: The ETG group demonstrated significant improvements in physical function (gait speed and stair climbing) and muscle strength (isokinetic strength and power in knee tests) $(p<.05)$ compared to the CTG group after 8 weeks. There were no significant differences for chair stand test $(p>.05)$ after 8 weeks.

CONCLUSIONS: Motorized eccentric-based training was more effective in improving muscle strength and physical function in aged individuals than conventional resistance training, which s suggests that eccentric training is may be more beneficial for older individuals to improve overall physical function.

Key words: Aging, Conventional resistance training, Motorized eccentric training, Physical performance

\section{INTRODUCTION}

Skeletal muscle function is the most important element of a healthy and active life in older adults and is closely related to every kind of physical effort [1]. Despite the natural phenomenon of losing muscle mass and strength in the aging process, this has recently been recognized as a disease and has been defined as sarcopenia [2]. The reason for this is that muscle atrophy due to aging is part of several functional disorders, including decreased muscle strength and flexibility, vulnerability to injury, and delayed functional recovery [3-5]. Compared to young and middleaged adults, people aged 70-85 years old experience a 30\% decrease in muscle mass [6].

Resistance training has become a strategy to prevent muscle atrophy [7]. It also improves physical function, muscle strength, and the ability to maintain muscle mass [8]. Recently, mechanism-based (power training, eccentric training, high intensity interval training, hybrid electrical stimulation training) interventions incorporated with age-related changes have been attempted in exercise interventions for older adults [9-12]. The age-

\footnotetext{
Corresponding author: Wook Song Tel +82-2-880-7791 Fax +82-2-872-2817 E-mail songw3asnu.ac.kr Keywords Aging, Conventional resistance training, Motorized eccentric training. Physical performance

Received 21 Jun 2019 Revised 22 Aug 2019 Accepted 29 Aug 2019
}

@) This is an Open Access article distributed under the terms of the Creative Commons Attribution Non-Commercial License (http://creativecommons.org/licenses/by-nc/4.0/) which permits unrestricted non-commercial use distribution, and reproduction in any medium, provided the original work is properly cited. 
related decrease in muscular strength depends on several types of muscle contractions [13]. Although the decrease in muscular strength appears in all types of muscle contractions with advanced age, the decrease in eccentric contractions is much less (10-20\%) compared with concentric contractions [14]. While this preservation of eccentric strength is a well-known phenomenon, the underlying mechanism has yet to be determined [14]. Therefore, considering the relatively preserved eccentric muscular strength, eccentric contractions can be a more efficient way for muscle strengthening in older adults [14]. For a given level of muscle contraction, eccentric contractions require only a small amount of energy consumption (less than that of isometric and shortening contractions) $[15,16]$. Eccentric contractions present a relatively low heart-lung burden [17]. In addition, eccentric activation is a core element for most functional movements commonly used in daily life [17]. Therefore, eccentric contractions could prove advantageous in groups such as older adults, heart-lung disease patients, and patients with chronic degenerative diseases [17]. Despite the advantages of eccentric training, these phenomena resulted in delayed onset muscle soreness (DOMS), but based on the elderly people so far, DOMS occurred less often than in young people [18]. This is because of physiological changes - older adults have a higher muscle stiffness with lower elasticity and lower proportion of type II muscle fibers which are more vulnerable to eccentric damage compared to young adults [5].

Despite the many advantages of eccentric training, conflicting research results are still being reported. In previous research, a study by Raj et al. [19] reported that eccentric contraction training had an effect on the stair climb test, timed up and go test, 6-m fast walk test, and isokinetic knee extensor strength, while the study conducted by Dias et al. [20] presented negative results due to conflicting ineffectiveness in the knee extension test, 6-m walk test, and stair climb test in older adults. Such prior work presents training of eccentric-biased contractions which did not induce pure eccentric movements [20]. This study did an eccentric training movement where concentric and eccentric motions were performed for 1.5 seconds and 4.5 seconds, respectively. For conventional resistance training, each motion was conducted for 1.5 seconds [20]. This is not seen as presenting the effect of pure eccentric contraction movement. Therefore, we will conduct a more precise eccentric-biased study by applying motorized eccentric movement to older adults.

The aim of this study was to investigate the effect of 8 weeks of pure motorization eccentric-based training on muscular strength and physical function in older adults, as compared to conventional resistance training. We hypothesized that 8 weeks of motorization eccentric training would improve physical function and muscular strength more than conventional resistance training.

\section{METHODS}

\section{Participants}

Participants admitted to elective clinical procedures at the $S$ university hospital and community-dwelling older adults from $\mathrm{S}$ city were screened by a blinded researcher at the baseline. Participants were considered to be included if they were older than 65 years of age and experienced no difficulties with independent walking or daily activities. The exclusion criteria included hypertension ( $\geq 150 / 90 \mathrm{mmHg}$ ), a score of less than 23 on the Mini-Mental State Examination (MMSE), diabetes, lower limb paralysis, a history of trauma, and cognitive impairment.

Of the participants, 10 men and 10 women (over the age of 65) agreed to participate in this study. Four ( 2 men and 2 women) were ineligible from participating when the study commenced due to the exclusion criteria. Thus, Eligible participants were randomly assigned in a 1:1 ratio to either the eccentric training group ( $\mathrm{n}=8,4$ men and 4women) or conventional resistance training group ( $\mathrm{n}=8,4$ men and 4women). The eight ETG group and CTG group participants received a complete training intervention from start to finish. This study was conducted from September 2015 to July 2017.

This study is a RCT study and the randomization sequence was prepared by blindly placing a pen on a random number table [21], with odd numbers allocated to the eccentric training group and even numbers allocated to the conventional resistance training group. The following data collection was conducted at two points: at the beginning to determine the baseline, and at the end of the 8th week (Fig. 1). The instructor explained to participants that they were not to allow in excessive physical activity the day before measurement. All tests were conducted at the same time (4:30 p.m.), and no food was taken one hour before the measurement.

\section{Study procedures}

The eccentric training group (ETG) and the conventional resistance training group (CTG) underwent training regimens consisting of either eccentric-based or seated leg press (Fig. 1). Before performing eccentric exercises, a pre-test was used to measure grip strength, muscular strength, and physical function. Interventions and follow-up evaluations were then completed (Fig. 1). Also, body weight and height were measured. For the body circumference, the waist, hip, and thigh measurements were taken 


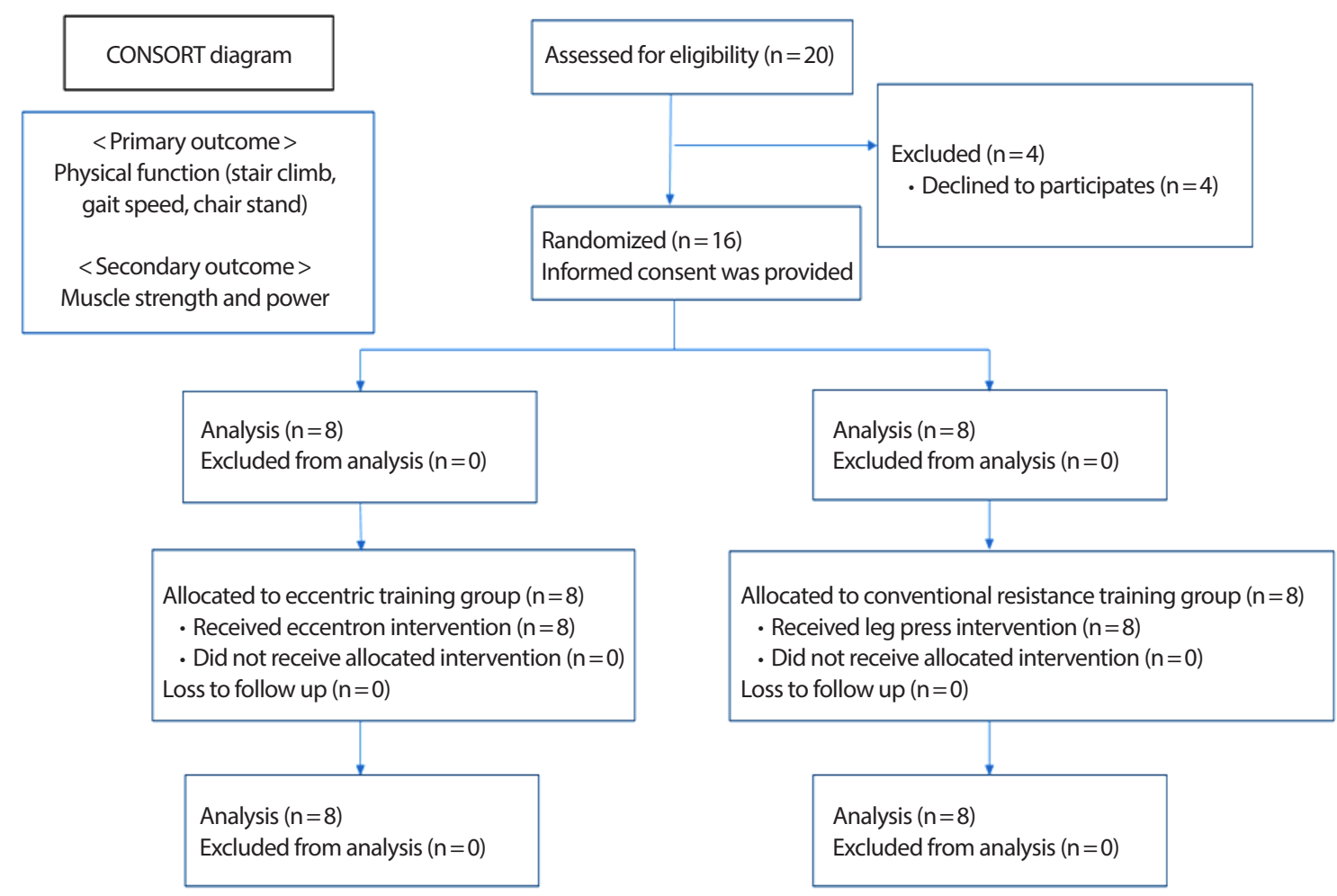

Fig. 1. CONSORT flow diagram of participants and allocation.

with a tapeline. Subjects were given the IPAQ. The IPAQ was used to compare the subject's physical activity before and after the intervention to evaluate the validity of all participants. To assess physical activity, we used the IPAQ short form protocol. The equation used to determine this value was: Total MET-min/week $=$ Walk $($ METs $\times \min \times$ days $)+$ Moderate $($ METs $\times$ min $\times$ days)+Vigorous (METs $\times \min \times$ days) [22]. These tests were selected because of typical components of the physical functions [20].

\section{Eccentric training protocol}

Prior to beginning the eccentric training program, an eccentric pre-test was examined in the motorized eccentric device by each participant. First, each participant sat on the motorized eccentric device and the seat and stride foot position were adjusted for elderly users. After entering the participant's personal information and pre-eccentric strength results, the participant began the main exercise using the motorized eccentric device. Eccentron ${ }^{\circledR}$ (BTE Tech., Hanover, MD, USA) (Fig. 2), which generates a pure eccentric motion. This program included 5-10 minutes of warm-up and 5 minutes of cool down (Table 1). The first week, participants adapted to the device by doing one 20-minute session twice a week at a speed of $18 \mathrm{rpm}$. For the subsequent 7 weeks, the protocol consisted of a 30-minute session twice a week for 8 weeks at a speed of $23 \mathrm{rpm}$. The training included two 2-minute

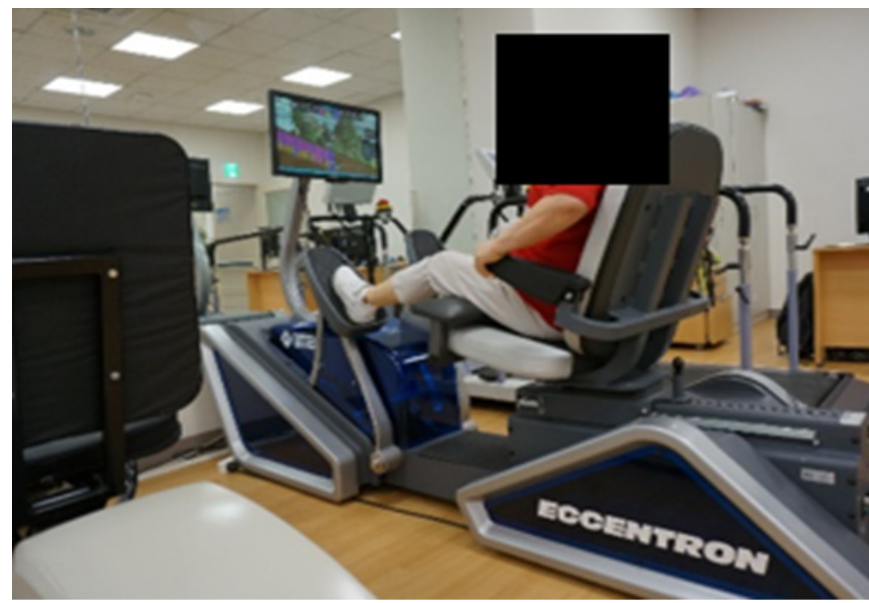

Fig. 2. Eccentric training device: negative resistance trainer.

rest periods. Training intensity was customized to each subject using 50\% of their maximal eccentric peak torque; $50 \%$ of eccentric peak torque is set by the device program using a pre-training test mode. For the advanced exercise mode, we measured the participants' strength prior to the exercise session and at the end of the second, fourth, and sixth weeks. The eccentric portion of the study focused on the quadriceps and gluteus maximus muscles. These exercises also focus on using an eccentric motion. As shown in Table 1, subjects performed conventional resistance training wherein different phases 
Table 1. Training Protocol

\begin{tabular}{|c|c|c|}
\hline Classification & ETG & CTG \\
\hline Warm-up (time) & Stretching (5-10 min) & Stretching (5-10 min) \\
\hline \multirow{7}{*}{ Main exercise } & Eccentric training & Leg press \\
\hline & Session duration (30 min) & Session duration (30 min) \\
\hline & $50 \%$ of estimated $1 \mathrm{RM}$ tested via the eccentric device & $50 \%$ of estimated 1 RM tested via the EN-Dynamic Seated Leg Press \\
\hline & Speed $23 \mathrm{reps} / \mathrm{min}$ (normal) & Phases: 1.5 sec press and pull \\
\hline & 1st week: 20 min (18 reps/min) & 1st week: 20 min \\
\hline & Weeks 2-8: $30 \mathrm{~min}$ & Weeks 2-8: $30 \mathrm{~min}$ \\
\hline & 2 times/week & 2 times/week \\
\hline Cool-down (time) & Stretching (5 min) & Stretching (5 min) \\
\hline
\end{tabular}

ETG, eccentric training group; CTG, conventional resistance training group.

(i.e., 1.5 seconds concentric phase and 4.5 seconds eccentric phase) of concentric and eccentric motion were included. This program consisted of three sets, with 10 repetitions for each set, three times per week [23,24].

\section{Conventional resistance training protocol}

The first week of the conventional resistance exercise program also consisted of two 20-minute training. The subsequent 7 weeks consisted of two 30-minute training per week. In this program, participants performed a general leg press that focused on the quadriceps. This training program also included 5-10 minutes of warm-up and 5 minutes of cool down. The intensity of the conventional resistance training was customized to each participant using 50\% of the estimated one-rep max (1RM) with an EN-Dynamic Seated Leg Press (Enraf-Nonius) (Fig. 3). All participants were encouraged to perform as many leg press repetitions as possible until they could no longer press or failed to complete another full range of motion. Participants performed knee resistance training in a sitting position with the foot placed on the center of the pedal of the EN-Dynamic machine. Participants were asked to fully extend and flex their knee joint from $90^{\circ}$ of knee flexion. Each action was rhythmically monitored by the researcher; the first second was spent extending the knee and the next second was spent flexing the knee. During the first week, the participant performed two sets at $50 \%$ of the estimated 1RM. For the advanced exercise mode, every 2 weeks, 1RM was retested, as was also done with the ETG. Rest intervals were the same as during eccentric training. Conventional resistance training also included homebased training focusing on concentric motion. As shown in Table 1, subjects performed conventional resistance training wherein equal phases (i.e., 1.5 seconds concentric phase and 1.5 seconds eccentric phase) of concentric and eccentric motion were included. This consisted of three sets, with 10 repetitions for each set, three times per week.

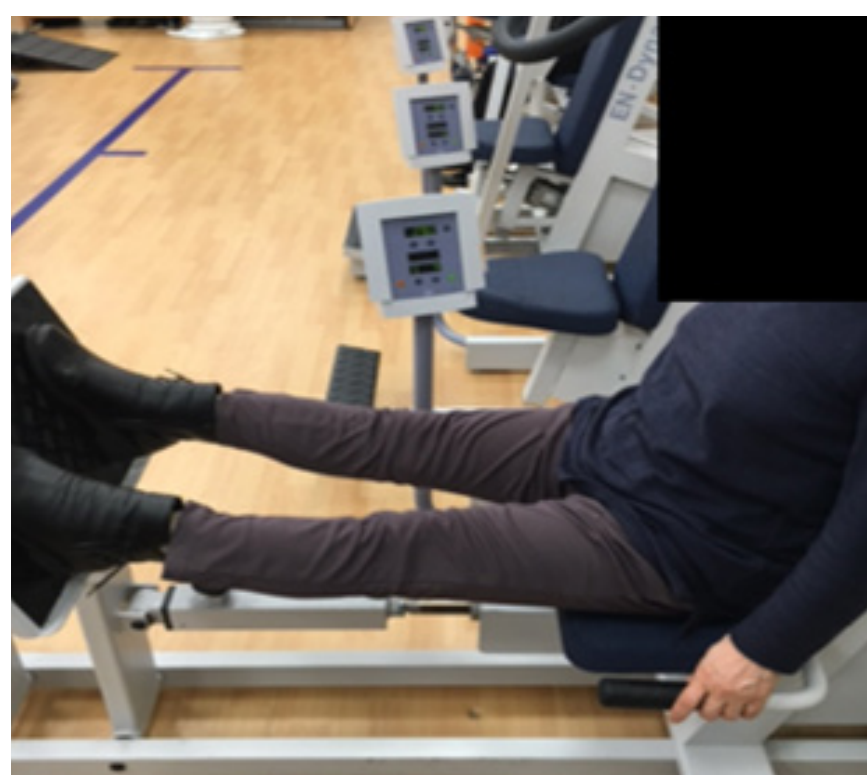

Fig. 3. EN-Dynamic Seated Leg Press.

\section{Main outcome measurement}

The physical function test included stair climbing, gait speed, and chair standing tests. For the stair climbing test, subjects were measured on eight $17-\mathrm{cm}$ stairs twice, requiring a step by step pattern, where the timer was activated when the first contact was made at the first step and ended when the contact occurred at the last step before and after the 8 weeks of exercise training. In the chair standing test, subjects had to get up from a chair measuring $41 \mathrm{~cm}$ in height with a flat seat. Subjects were asked to stand up and sit down 5 times as quickly as possible with their arms folded across the chest; they stood up until full extension was observed at the trunk and lower limb joints, and returned to a seated position with their back fully supported at the back of the chair. To determine gait speed, an examiner used a stopwatch to time how long it took subjects to walk along a marked tape. The Kinect (Korea Institute of Machinery and Materials) device was used to measure gait speed. 


\section{Secondary outcome measurement}

Isometric peak torque was measured using a Baltimore Therapeutic Equipment (BTE) Primus RS (BTE Tech., Hanover, MD, USA). The subject was asked to sit on the treatment chair and a standard stabilization strap was placed on the upper ankle. The knee was kept at $90^{\circ}$ flexion, and the foot was positioned in dorsi flexion. The subject's hands were placed on the edge of the side of the chair and the trunk, hips, and midthigh were stabilized in the chair by the straps. Three repeated isometric strength trials were performed at each load, then the average was plotted.

The isokinetic test used the same device as the isometric test, and the subject performed a $60 \% \mathrm{sec}$ knee flexion and extension five times. Each isokinetic contraction was performed through a full range of motion. Before each subject began the isokinetic test, the subject warmed up using a 50-60\% knee extension/flexion once.

To measure power, the test used the same machine as the isometric and isokinetic tests. The ankle was placed in a neutral position, and the subjects performed a range of motion for 10 seconds, once. After the warm up, knee extension and flexion were performed as quickly as possible during a period of 10 seconds. During the testing session, subjects were given verbal encouragement to help ensure maximal effort.

\section{Statistical analyses}

The sample size of this study consisted of eight people in each group, as determined by the $G$ power 3.10 program. A previous study [25] showed a

Table 2. Baseline evaluation of body composition and body circumference

\begin{tabular}{lrrr}
\hline Measures & $\begin{array}{c}\text { Eccentric train- } \\
\text { ing group }(\mathrm{n}=8) \\
\text { Mean (SD) }\end{array}$ & $\begin{array}{c}\text { Conventional re- } \\
\text { sistance training } \\
\text { group }(\mathrm{n}=8) \\
\text { Mean (SD) }\end{array}$ & p-value \\
\hline Body composition & & & \\
Age (yr) & $72(2.62)$ & $75(1.41)$ & \\
Height (cm) & $162.74(2.24)$ & $157.25(8.04)$ & .164 \\
Body weight (kg) & $68.33(9.60)$ & $60.11(7.26)$ & .092 \\
Skeletal muscle mass (kg) & $25.71(1.30)$ & $22.23(1.04)$ & .063 \\
Muscle mass/BW (\%) & $0.37(0.01)$ & $0.36(0.01)$ & .598 \\
BMl (kg/m²) & $26.07(1.37)$ & $24.10(1.54)$ & .434 \\
Fat $(\%)$ & $31.40(2.93)$ & $31.91(3.36)$ & .921 \\
WHR (\%) & $0.89(0.02)$ & $0.90(0.03)$ & .665 \\
Body circumference & & & \\
Rt thigh (cm) & $48.18(4.03)$ & $47.25(2.01)$ & .229 \\
Lt thigh (cm) & $48.12(4.01)$ & $47.06(2.11)$ & .132 \\
Waist (cm) & $93.62(9.05)$ & $88.43(6.31)$ & .121 \\
Hip (cm) & $99.56(7.18)$ & $94.00(3.16)$ & .068 \\
\hline
\end{tabular}

$\mathrm{BMI}$, body mass index; WHR, waist to hip ratio; Rt, right; Lt, left. significant difference in maximal voluntary contraction in two groups of healthy older adult subjects $(\mathrm{n}=16, p=.017)$. Results are expressed as the mean \pm standard deviation (SD). We determined the effect sizes (Cohen's $d$ ) for measures where $d$ is defined as the difference between the adjusted means of two interventions divided by the pooled SD of those means. Sample size was measured using the F-test and ANOVA between factors, and a significant difference was based on 0.05 . As a result, the critical $\mathrm{F}$ value was 3.49 , the total sample size needed was at least 16 subjects, and the actual power was 0.8056 . In this study, we recruited 20 subjects to allow for a 20\% drop-out rate; therefore, the total sample size was 16 subjects.

The analysis for normality will be confirmed using the KolmogorovSmirnov test, given that it is appropriate when used with a small sample size [26]. A two-way repeated measures ANOVA, followed by a LSD post hoc test, as used to assess any differences between groups after the intervention. The level of significance was set at $p<.05$. Statistical analysis was performed using SPSS software version 21.0 (SPSS Inc., Chicago, IL, USA).

Table 3. Changes in exercise on outcome variables from baseline to 8 weeks follow-up

\begin{tabular}{lccc}
\hline Outcome variable & $\begin{array}{c}\text { Baseline mean } \\
\text { (SD) }\end{array}$ & $\begin{array}{c}\text { 8th week mean } \\
\text { (SD) }\end{array}$ & $p$-value \\
\hline Stair climb (sec) & & & \\
ETG & $4.65(0.29)$ & $3.98(0.14)$ & .002 \\
CTG & $4.71(0.26)$ & $4.56(0.13)$ & .380 \\
Gait speed (sec) & & & \\
ETG & $4.16(0.50)$ & $3.46(0.26)$ & .002 \\
CTG & $4.09(0.28)$ & $3.94(0.12)$ & .067 \\
Chair stand (sec) & & & \\
ETG & $10.25(0.95)$ & $9.23(0.71)$ & .013 \\
CTG & $10.44(0.84)$ & $9.91(0.63)$ & .040 \\
Isometric (nm) & & & \\
ETG & $113.17(7.49)$ & $137.66(6.78)$ & .000 \\
CTG & $121.79(9.24)$ & $130.08(8.70)$ & .000 \\
Isokinetic (nm) & & & \\
ETG & $74.33(6.86)$ & $86.46(7.31)$ & .000 \\
CTG & $78.93(7.78)$ & $81.72(7.52)$ & .181 \\
Power (watts) & & & \\
ETG & $64.95(8.14)$ & $70.60(7.60)$ & .015 \\
CTG & $66.29(8.89)$ & $69.63(7.61)$ & .092 \\
Grip strength (kg) & & & .170 \\
ETG & $27.05(2.05)$ & $28.20(2.00)$ & .106 \\
CTG & $23.42(1.86)$ & $24.26(1.79)$ & .158 \\
IPAQ (MET-min/week) & & & \\
ETG & $887.14(93.99)$ & $917.12(70.49)$ & .172 \\
CTG & $800.99(83.95)$ & $842.75(88.24)$ & .117 \\
\hline
\end{tabular}

ETG, ecentric training group; CTG, conventional resistance training group; IPAQ, International Physical Activity Questionnaire. 


\section{RESULTS}

\section{Baseline evaluation}

Statistical analysis was performed with eight subjects in the eccentric exercise group (age, $72.38 \pm 2.62$ years; weight, $67.76 \pm 9.12 \mathrm{~kg}$; height, $162.66 \pm 2.21 \mathrm{~cm})$ and eight subjects in the conventional resistance exercise group (age, $75.0 \pm 1.41$ years; weight, $59.63 \pm 7.08 \mathrm{~kg}$; height, 157.17 \pm $8.39 \mathrm{~cm}$ ). There were no significant differences in subject characteristics with respect to time, group and the interaction between time and group ( $p>.05$ ) (Table 2). None of the variables related to the IPAQ significantly changed after the interventions $(887.14 \pm 93.99$ vs. $917.12 \pm 70.49$ MET$\min /$ week) $(p>.05)$ (Table 3).

\section{Comparison of physical performance}

The result of the stair climb test represented a significant improvement in time $(p<.001)$, group $(p<.01)$ and the interaction between time and group $(p<.05)(4.655 \pm 0.298$ vs. $3.989 \pm 0.149$, respectively) (Fig. 4). There were significant changes in gait speed with respect to time $(p<.001)$, group $(p<.01)$ and the interaction between time and group $(p<.05)$. The gait speed ability in the ETG was significantly decreased compared to the pre-test ( $4.164 \pm 0.059$ vs. $3.465 \pm 0.266$, respectively, $p<.01)$. However, there was no significant difference between the pre- and post-tests in the CTG (4.098 \pm 0.289 vs. $3.945 \pm 0.125$, respectively, $p>.05$ ) (Fig. 4). The effect size for stair climb and gait speed was 0.19 and 0.15 , respectively. No

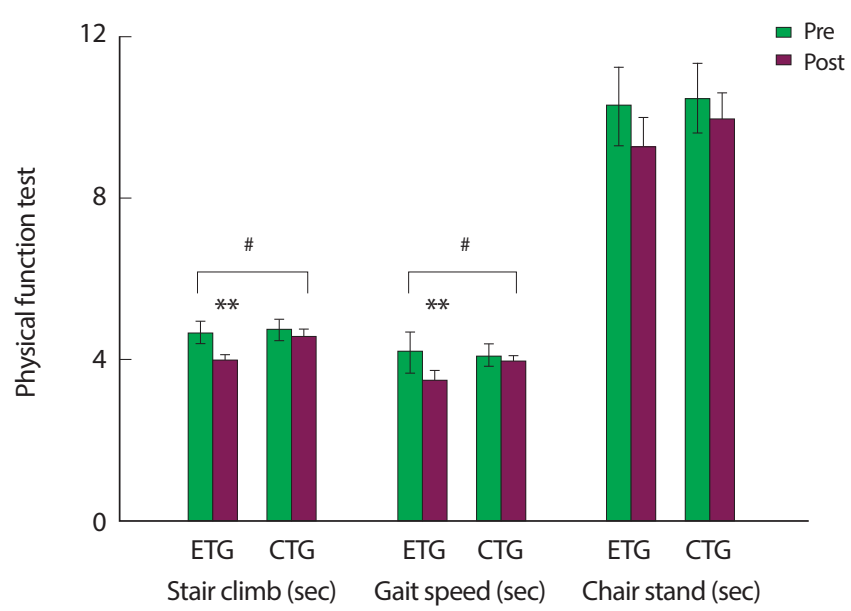

Fig. 4. Stair climb and gait speed test resulits before and after two different training programs. ETG, eccentric training group; CTG, conventional resistance training group. ${ }^{*} p<.05$ by using the least significant difference (LSD) test post hoc to analyze differences between the ETG and CTG. ${ }^{* *} p<.01$ by using the LSD test post hoc to analyze differences between pre- and posttraining. significant changes were observed in chair stand with the interaction between time and group $(p>.05)$.

\section{Comparison of secondary outcome variables}

The results showed an increase in isokinetic strength in both the ETG and CTG (pre-ETG vs. post-ETG, $77.587 \pm 6.836$ vs. $98.308 \pm 4.04, p<.01$ and pre-CTG vs. post-CTG, $71.418 \pm 6.071$ vs. $82.179 \pm 3.677$ ) (Fig. 5). The results showed a significant improvement in the ETG (pre-ETG vs. post-ETG, $66.817 \pm 4.083$ vs. $78.316 \pm 3.988, p<.05)$. In addition, there was a significant difference between the ETG and CTG $(78.316 \pm 3.988$ vs. $64.734 \pm 5.947$, respectively, $p<.05$ ) (Fig. 5). The effect size for isokinetic strength and power was 0.63 and 0.13 , respectively. No significant changes were observed in isometric knee strength with the interaction between time and group ( $p>.05)$ (Fig. 5).

\section{DISCUSSION}

This is the randomized controlled investigation of the effect of lower extremity exercise training in healthy older adults. We found that the results of the present study characterized that motorized eccentric training yielded benefits concerning stair climbing, gait speed, and chair stand in the physical function of the primary outcome, in addition to isometric and isokinetic muscle strength of the secondary outcome in this population of older adults.

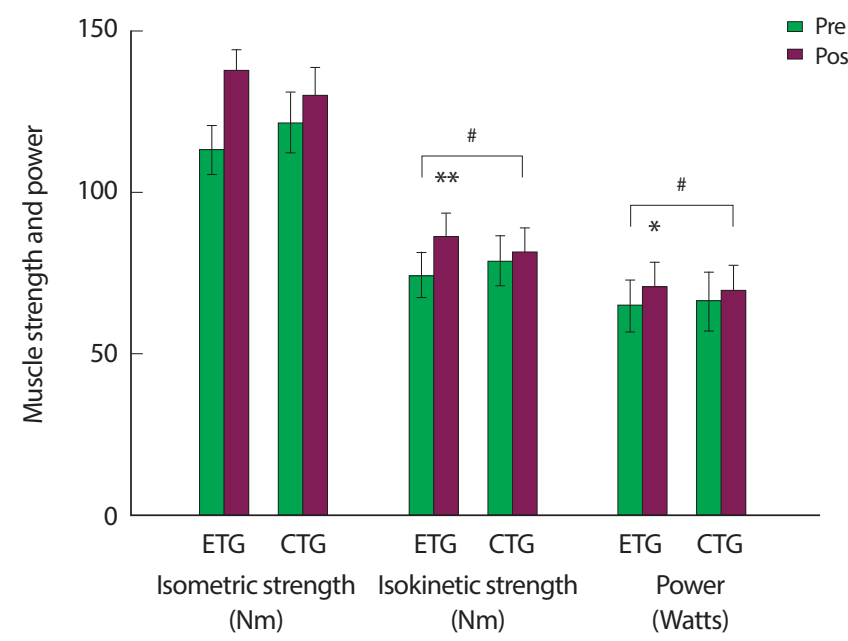

Fig. 5. Isometric strength, isokinetic strength $(60 \% \mathrm{sec})$ and power before and after two different training programs. ETG, eccentric training group; CTG, conventional resistance training group. ${ }^{*} p<.05$ by using the LSD test post hoc to analyze the differences between the ETG and CTG. ${ }^{* *} p<.01$, ${ }^{*} p<.05$ by using the LSD test post hoc to analyze differences between preand post-training. 
A recent previous study [20,27-30] showed that an eccentric exercise was conducted and it was applied to the exercise by applying eccentric movement to existing concentric. However, the result of these studies were very controversial. Gault et al. [27] Chen et al. [28], and Symons et al. [29] reported several positive effects such as functional capacity and knee strength of eccentric exercise interventions in older adults. But, a study that compared conventional resistance training with eccentric found that they were not effective for improving knee strength and functional capacity $[20,30]$. The point of above study was allowed to concentric movement when doing the eccentric motion in the leg. Therefore, we utilized a specialized device using motorization, and applied it to elderly people over 65 years old. Also, we have conducted test to make more accurate eccentric studies in older adults.

In this study, we observed improvements in functional performance tests and knee extension muscular strength in both groups after eight weeks of training. Studies have shown a rapid increase in maximum strength after eight weeks of motorized eccentric training [20,31,32]. In this study, we did not investigate how much time is required for muscle adaptation with eccentric or conventional resistance training; however, our findings suggest that eight weeks should be adequate with both types of exercise to significantly impact strength and functional capacity in elderly subjects [33].

In this study, no significant difference was found between the ETG and CTG regarding isometric knee strength. However, in the ETG, a significant increase in isokinetic knee extension following the exercise period. Additionally, there was significant difference in isotonic knee power in the ETG after the exercise period. These results suggest that both types of exercise can increase muscular strength, but eccentric exercise is better effective in older adults [14].

The functional tests used in this study are closely related to the stair climbing, gait speed, and chair stand tests [20]. A number of tasks have a high risk of fall in elderly people; therefore, functional capacity seems to be more important than strength in this population. The majority of exercises to reduce the risk of fall, such as stair descent, are performed eccentrically [5]. Thus, the assessment of eccentric tasks should be the primary focus when evaluating the potential benefits of an eccentric exercise program [20].

The strength and functionality values found in this study resemble those found in previous studies on knee extensions [34], leg presses [35], and chair stand tests $[36,37]$. Furthermore, in our study, participants in the motorized eccentric training group completed the stair climbing test in a shorter time than did participants in the conventional resistance exercise group [35-37]. Previous studies have shown that gains in muscular strength and mass in healthy adults aged 18 to 65 years are higher in an exclusively eccentric exercise program compared with an exclusively concentric exercise program [38]. However, Symons' study showed that the eccentric exercise group did not demonstrate superior gains in voluntary strength compared with the isometric and concentric groups [29]. In addition, Dias et al. [20] only observed significant improvement during the first 6 of 12 weeks in 1RM knee extension strength and functional tests [39].

It is important to mention that we matched the progression of exercise intensity between groups using the results of the pre-strength test, which was the announced pre-strength test from the motorized eccentric device (BTE ${ }^{\mathrm{TM}}$ Inc.), and $50 \%$ of eccentric peak torque is set by the device program through a pre-training test mode during the baseline evaluation. In addition, from the pre-strength test using the motorized eccentric device (BTE ${ }^{\mathrm{TM}}$ Inc.), we set the exercise target zone at 50\% of full strength for each person. Likewise, for subjects in the conventional resistance training group, we measured their estimated 1RM by an EN-Dynamic Seated Leg Press.

Despite these limitations, our results provide a preliminary indication that a motorized eccentric exercise program may be effective at improving physical function and muscle strength, and conventional resistance training can also improve some aspects of physical function and strength in healthy older adults. Motorized eccentric training was more beneficial for physical function and muscle strength compared to conventional resistance training. However, some other variables, such as the isotonic power or body circumference, were not more improved in the ETG that those in the CTG. These findings may be different in a trial with a larger sample size.

Further research is needed to determine whether longer periods of training (more than 8 weeks) or if having more groups could demonstrate differences between motorized eccentric and conventional training more clearly.

The present study has some limitations that should be noted. First, the physical conditions or personal physical fitness levels of study participants may differ, which can affect the outcome. Second, the results of this study may not be generalizable due to small sample size used. Therefore, a study using a larger sample is required. Third, the two types of exercise intervention may differ in terms of intensity and volume, although we attempted to match them as much as possible with regard to the frequency and duration of intervention. Despite the study limitations, there is a novelty that we could suggest with this study. 1) Although most of the previous 
studies included concentric motion during eccentric training, our study has been done based on a specialized device using motorized eccentric motion, which means that the instructor led the participants to concentrate solely on eccentric movement during their intervention period. 2) In addition, while the previous studies mostly focused on muscle strength, but our study could highlight the change of muscle power variables such as stair climbing test and gait speed, and chair stand test following eccentric training.

\section{CONCLUSION}

The unique findings of this study are that motorized lower limb eccentric training is feasible, and is effective as a form of training modality. Over an 8-week intervention period, eccentric training resulted in better outcomes (isokinetic and isometric muscle strength, gait speed, stair climb, chair stand) by contrast with conventional resistance training for older adults. Lastly, motorized eccentric training is a potentially beneficial training modality that can be applied to older adults for the sake of functional ability and muscle strength.

\section{ACKNOWLEDGEMENT}

This research was supported by Basic Science Research Program through National Research Foundation of Korea (NRF) funded by the Ministry of Education (NRF-2016R1D1A1B03935518) and Seoul National University Bundang Hospital Fund (SNUBH-04-2011-005).

\section{CONFLICT OF INTEREST}

No potential conflict of interest relevant to this article was reported.

\section{REFERENCES}

1. Trombetti A, Reid KF, Hars M, Herrmann FR, Pasha E, et al. Age-associated declines in muscle mass, strength, power, and physical performance: impact on fear of falling and quality of life. Osteoporos Int. 2016;27(2):463-71.

2. Doherty TJ. Invited review: Aging and sarcopenia. J Appl Physiol (1985). 2003;95(4):1717-27.

3. Frontera WR, Reid KF, Phillips EM, Krivickas LS, Hughes VA, et al. Muscle fiber size and function in elderly humans: a longitudinal study.
J Appl Physiol (1985). 2008;105(2):637-42.

4. Krivickas LS, Fielding RA, Murray A, Callahan D, Johansson A, et al. Sex differences in single muscle fiber power in older adults. Med Sci Sports Exerc. 2006;38(1):57-63.

5. Lim JY. Therapeutic potential of eccentric exercises for age-related muscle atrophy. Integr Med Res. 2016;5(3):176-81.

6. Shafiee G, Keshtkar A, Soltani A, Ahadi Z, Larijani B, et al. Prevalence of sarcopenia in the world: a systematic review and meta- analysis of general population studies. J Diabetes Metab Disord. 2017;16:21.

7. Yoshiko A, Tomita A, Ando R, Oqawa M, Kondo S, et al. Effects of 10-week walking and walking with home-based resistance training on muscle quality, muscle size, and physical functional tests in healthy older individuals. Eur Rev Aging Phys Act. 2018;15:13.

8. Mangione KK, Miller AH, Naughton IV. Cochrane review: Improving physical function and performance with progressive resistance strength training in older adults. Phys Ther. 2010;90(12):1711-5.

9. Alcazar J, Losa-Reyna J, Rodriquez-Lopez C, Alfaro-Acha A, Rodriquez-Manas L, et al. The sit-to-stand muscle power test: An easy, inexpensive and portable procedure to assess muscle power in older people. Exp Gerontol. 2018;112:38-43.

10. Abdel-Aziem AA, Soliman ES, Abdelraouf OR. Isokinetic peak torque and flexibility changes of the hamstring muscles after eccentric training: Trained versus untrained subjects. Acta Orthop Traumatol Turc. 2018;52(4):308-14.

11. Abderrahman AB, Rhibi F, Ouerghi N, Hackney AC, Saeidi A, et al. Effects of recovery mode during high intensity interval training on glucoregulatory hormones and glucose metabolism in response to maximal exercise. J Athl Enhanc. 2018;7(3).

12. Taylor JA, Picard G, Porter A, Morse LR, Pronovost MF, et al. Hybrid functional electrical stimulation exercise training alters the relationship between spinal cord injury level and aerobic capacity. Arch Phys Med Rehabil. 2014;95(11):2172-9.

13. Kalyani RR, Corriere M, Ferrucci L. Age-related and disease-related muscle loss: the effect of diabetes, obesity, and other diseases. Lancet Diabetes Endocrinol. 2014;2(10):819-29.

14. Roig M, Macintyre DL, Eng JJ, Narici MV, Maganaris CN, et al. Preservation of eccentric strength in older adults: Evidence, mechanisms and implications for training and rehabilitation. Exp Gerontol. 2010; 45(6):400-9.

15. Enoka RM. Eccentric contractions require unique activation strategies by the nervous system. J Appl Physiol (1985). 1996;81(6):2339-46. 
16. Tesch PA, Dudley GA, Duvoisin MR, Hather BM, Harris RT. Force and EMG signal patterns during repeated bouts of concentric or eccentric muscle actions. Acta Physiol Scand. 1990;138(3):263-71.

17. Lollo PC, Moura CS, Morato PN, Amaya-Farfan J. Differential response of heat shock proteins to uphill and downhill exercise in heart, skeletal muscle, lung and kidney tissues. J Sports Sci Med. 2013;12(3): 461-6.

18. Lavender AP, Nosaka K. Comparison between old and young men for changes in makers of muscle damage following voluntary eccentric exercise of the elbow flexors. Appl Physiol Nutr Metab. 2006;31(3): 218-25.

19. Raj IS, Bird SR, Westfold BA, Shield AJ. Effects of eccentrically biased versus conventional weight training in older adults. Med Sci Sports Exerc. 2012;44(6):1167-76.

20. Dias CP, Toscan R, de Camargo M, Pereira EP, Griebler N, et al. Effects of eccentric-focused and conventional resistance training on strength and functional capacity of older adults. Age (Dordr). 2015; 37(5):99.

21. Altman DG, Bland JM. Statistics notes. Treatment allocation in controlled trials: why randomise? BMJ 1999;318(7192):1209.

22. Ibrahim N, Moy FM, Awalludin IA, Ali Z, Ismail IS. The health-related quality of life among pre-diabetics and its association with body mass index and physical activity in a semi-urban community in Malaysia--a cross sectional study. BMC Public Health. 2014;14:298.

23. LaStayo P, Marcus R, Dibble L, Wong B, Pepper G. Eccentric versus traditional resistance exercise for older adult fallers in the community: a randomized trial within a multi-component fall reduction program. BMC Geriatr. 2017;17(1):149.

24. Gluchowski A, Harris N, Dulson D, Cronin J. Chronic eccentric exercise and the older adult. Sports Med. 2015;45(10):1413-30.

25. Vaczi M, Nagy SA, Koszegi T, Ambrus M, Bogner P, et al. Mechanical, hormonal, and hypertrophic adaptations to 10 weeks of eccentric and stretch-shortening cycle exercise training in old males. Exp Gerontol. 2014;58:69-77.

26. Leone N, Courbon D, Berr C, Barberger-Gateau P, Tzourio C, et al. Abdominal obesity and late-onset asthma: cross-sectional and longitudinal results: the 3C study. Obesity (Silver Spring). 2012;20(3):628-35.

27. Gault ML, Willems ME. Aging, functional capacity and eccentric exercise training. Aging Dis. 2013;4(6):351-63.

28. Chen TC, Tseng WC, Huang GL, Chen HL, Tseng KW, et al. Superior effects of eccentric to concentric knee extensor resistance training on physical fitness, insulin sensitivity and lipid profiles of elderly men. Front Physiol. 2017;8:209.

29. Symons TB, Vandervoort AA, Rice CL, Overend TJ, Marsh GD. Effects of maximal isometric and isokinetic resistance training on strength and functional mobility in older adults. J Gerontol A Biol Sci Med Sci. 2005;60(6):777-81.

30. Gault ML, Clements RE, Willems ME. Functional mobility of older adults after concentric and eccentric endurance exercise. Eur J Appl Physiol. 2012;112(11):3699-707.

31. Cadore EL, Rodriguez-Manas L, Sinclair A, Izquierdo M. Effects of different exercise interventions on risk of falls, gait ability, and balance in physically frail older adults: a systematic review. Rejuvenation Res. 2013;16(2):105-14.

32. Hakkinen K, Pakarinen A. Serum hormones and strength development during strength training in middle-aged and elderly males and females. Acta Physiol Scand. 1994;150(2):211-9.

33. Pinto RS, Correa CS, Radaelli R, Cadore EL, Brown LE, et al. Shortterm strength training improves muscle quality and functional capacity of elderly women. Age (Dordr). 2014;36(1):365-72.

34. Rice DA, McNair PJ, Lewis GN, Mannion J. Experimental knee pain impairs submaximal force steadiness in isometric, eccentric, and concentric muscle actions. Arthritis Res Ther. 2015;17:259.

35. Hanson ED, Srivatsan SR, Agrawal S, Menon KS, Delmonico MJ, et al. Effects of strength training on physical function: influence of power, strength, and body composition. J Strength Cond Res. 2009;23(9): 2627-37.

36. Peiffer JJ, Galvao DA, Gibbs Z, Smith K, Turner D, et al. Strength and functional characteristics of men and women 65 years and older. Rejuvenation Res. 2010;13(1):75-82.

37. Tiedemann A, Shimada H, Sherrington C, Murray S, Lord S. The comparative ability of eight functional mobility tests for predicting falls in community-dwelling older people. Age Ageing. 2008;37(4):430-5.

38. Roig M, O’Brien K, Kirk G, Murray R, McKinnon P, et al. The effects of eccentric versus concentric resistance training on muscle strength and mass in healthy adults: a systematic review with meta-analysis. $\mathrm{Br}$ J Sports Med. 2009;43(8):556-8.

39. Cadore EL, Izquierdo M, Pinto SS, Alberton CL, Pinto RS, et al. Neuromuscular adaptations to concurrent training in the elderly: effects of intrasession exercise sequence. Age (Dordr). 2013;35(3):891-903. 